\title{
ON RIESZ SUMMABILITY OF FOURIER SERIES BY EXPONENTIAL MEANS
}

\author{
FU TRAING WANG
}

Let $f(t)$ be an integrable periodic function with the period $2 \pi$. Let its Fourier series be

$$
f(t) \sim \frac{a_{0}}{2}+\sum_{n=1}^{\infty} a_{n} \cos n t+b_{n} \sin n t
$$

and let

$$
\begin{aligned}
\phi(t) & =\{f(x+t)+f(x-t)-2 s\} / 2, \\
\phi_{\beta}(t) & =(1 / \Gamma(\beta)) \int_{0}^{t}(t-u)^{\beta-1} \phi(u) d u, \\
A_{n} & =a_{n} \cos n x+b_{n} \sin n x .
\end{aligned}
$$

We shall prove the following result. ${ }^{1}$

$$
\text { If } A_{n}>-K n^{-\beta / \gamma}(\gamma>\beta>0) \text { and }
$$

$$
\phi_{\beta}(t)=o\left(t^{\gamma}\right)
$$

the Fourier series (1) converges to $s$ at $t=x$.

Set $\alpha=1-\beta / \gamma$, and

$$
C_{\tau}(\omega)=a_{0} e^{\tau \omega^{\alpha}} / 2+\sum_{n<\omega}\left(e^{\omega^{\alpha}}-e^{n^{\alpha}}\right)^{\tau} A_{n}
$$

The Fourier series (1) is said to be summable $\left(e^{n^{\alpha}}, \tau\right)$ to the sum $s$ if $^{2}$

$$
C_{\tau}(\omega)=s e^{\tau \omega^{\alpha}}+o\left(e^{\tau \omega^{\alpha}}\right) \quad \text { as } \omega \rightarrow \infty .
$$

Concerning this kind of summability we have the following theorem.

THEOREM. $^{3}$ If (2) holds and $\tau$ is a positive integer greater than $\gamma+1$ the Fourier series (1) is summable $\left(e^{n^{\alpha}}, \tau\right)$ to the sum $s$ at $t=x$.

Received by the editors January 6, 1944 .

1 G. H. Hardy and J. E. Littlewood [2], F. T. Wang [6]. Numbers in brackets refer to the references listed at the end of the paper.

2 G. H. Hardy and M. Riesz [3].

3 Under the hypotheses of the Theorem I have established that the Fourier series (1) is summable $\left(e^{n \alpha}, \gamma+\delta\right)(\delta>0)$ to the sum $s$ at $t=x$, but the proof is very complicated. See F. T. Wang [6]. 
The convergence criterion above is deducible from this theorem by the use of a result of Hardy, ${ }^{4}$ namely if the series $\sum_{n=0}^{\infty} a_{n}$, with terms $a_{n} \geqq-K n^{\alpha-1}, 0<\alpha<1$, is summable $\left(e^{n^{\alpha}}, \tau\right)$, it is convergent.

To prove the theorem we write

$$
E_{\tau}(\omega, t)=\tau \alpha \int_{0}^{\omega}\left(e^{\omega^{\alpha}}-e^{x^{\alpha}}\right)^{\tau-1} e^{x^{\alpha}} x^{\alpha-1} \frac{\sin x t}{t} d x .
$$

Then we have ${ }^{5} C_{\tau}(\omega)=(2 / \pi) \int_{0}^{1} \phi(t) E_{\tau}(\omega, t) d t+s e^{r \omega^{\alpha}}+o\left(e^{\tau \omega^{\alpha}}\right)$, and if we take $\omega_{1}=2^{-1 / \alpha} \omega$, then

$$
\begin{aligned}
E_{\tau}(\omega, t) & =\alpha \tau \int_{\omega 1}^{\omega}\left(e^{\omega^{\alpha}}-e^{x^{\alpha}}\right)^{r-1} e^{x^{\alpha}} x^{\alpha-1} \frac{\sin x t}{t} d x+o\left(e^{\tau \omega^{\alpha}}\right) \\
& =F_{\omega}(t)+o\left(e^{r \omega^{\alpha}}\right) .
\end{aligned}
$$

Hence

$$
C_{\tau}(\omega)=(2 / \pi) \int_{0}^{1} \phi(t) F_{\omega}(t) d t+s e^{\tau \omega^{\alpha}}+o\left(e^{\tau \omega^{\alpha}}\right) .
$$

By setting $n=[\beta]+1$ and differentiating under the integral sign we get

$$
\begin{aligned}
F_{\omega}^{(n)}(t) & =\frac{d^{n}}{d t^{n}}\left\{\alpha \tau \int_{\omega_{1}}^{\omega}\left(\omega^{\omega^{\alpha}}-e^{x^{\alpha}}\right)^{r-1} e^{x^{\alpha}} x^{\alpha-1} \frac{\sin x t}{t} d x\right\} \\
& =\sum_{i=0}^{n} K_{i} \int_{\omega_{1}}^{\omega}\left(e^{\omega^{\alpha}}-e^{x^{\alpha}}\right)^{r-1} e^{x^{\alpha}} x^{\alpha-1+n-i} \frac{\sin (x t-a)}{t^{i+1}} d x
\end{aligned}
$$

where $a=(n-i) \pi / 2$.

By mathematical induction we can easily establish the formula

$$
\begin{aligned}
& \left(d^{r} / d x^{r}\right)\left\{\left(e^{\omega^{\alpha}}-e^{x^{\alpha}}\right)^{r-1} e^{x^{\alpha}} x^{\alpha-1+n-i}\right\} \\
& =\sum_{j=1}^{r} \sum_{p=0}^{r} K_{i p} e^{(r-j) \omega^{\alpha}} e^{j x^{\alpha}} x^{(p+1)(\alpha-1)+n-i-(r-p)} .
\end{aligned}
$$

Then by the use of (5) and (6) and an integration by parts we find

$$
\begin{aligned}
F_{\omega}^{(n)}(t)= & \sum_{i=0}^{n} \sum_{j=1}^{\tau} \sum_{p=0}^{\tau-1} K_{i j p} e^{(\tau-j) \omega^{\alpha}} \int_{\omega_{1}}^{\omega} e^{j x^{\alpha}} x^{c} \frac{\sin (x t-b)}{t^{i+\tau}} d x \\
& +O\left(e^{\left.(\tau-1 / 2) \omega^{\alpha} \omega^{k_{1}} t^{-k 2}\right),}\right.
\end{aligned}
$$

4 G. H. Hardy [4].

F F. T. Wang [6].

6 Throughout this paper we use $K$ or $K_{i} \cdots$ as a constant different in different occurrences. 
where $c=(p+1)(\alpha-1)+n-i-(\tau-1-p)$, and $b=(n-i-\tau+1) \pi / 2$.

Put $t=1$ in $(7)$. Then $F_{\omega}^{(m)}(0)$ is finite for $1 \leqq m<n$, and $F_{\omega}^{(m)}(1)$ $=o\left(e^{\tau \omega^{\alpha}}\right)$. Successive integration of (4) by parts yields

$$
C_{\tau}(\omega)=(2 / \pi) \int_{0}^{1} \phi_{n}\left(t F_{\omega}^{(n)}(t) d t+s e^{\tau \omega^{\alpha}}+o\left(e^{\tau \omega^{\alpha}}\right) .\right.
$$

By a theorem on the fractional integral ${ }^{7}$

$$
\phi_{n}(t)=(1 / \Gamma(n-\beta)) \int_{0}^{t}(t-u)^{n-\beta-1} \phi_{\beta}(u) d u,
$$

we have

$$
C_{\tau}(\omega)=(2 / \pi) \int_{0}^{1} \phi_{\beta}(u) H_{\omega}(u) d u+s e^{\tau \omega^{\alpha}}+o\left(e^{\tau \omega^{\alpha}}\right),
$$

where

$$
\begin{array}{rlr}
H_{\omega}(u)= & (1 / \Gamma(n-\beta)) \int_{u}^{1}(t-u)^{n-\beta-1} F_{\omega}^{(n)}(t) d t & (n>\beta>n-1) \\
& =F_{\omega}^{(n)}(u) & (n=\beta) .
\end{array}
$$

Concerning $H_{\omega}(u)$ we require the following two lemmas.

Lemma 1. For $\omega>K$ and $0<u<1$,

$$
\begin{aligned}
H_{\omega}(u)= & \sum_{i=0}^{n-1} O\left(e^{\tau \omega^{\alpha}} \omega^{\beta-i} u^{-i-1}\right)+\sum_{i=0}^{n-1} O\left(e^{\tau \omega^{\alpha}} \omega^{n-i-1} u^{n-\beta-i-2}\right) \\
& +O\left(e^{\tau \omega^{\alpha} \omega^{n-1}}(1-u)^{n-\beta-1}\right)+O\left(e^{\tau \omega^{\alpha}} u^{-\beta-1}\right) .
\end{aligned}
$$

Proof. From (10) and (5) we have

$$
\begin{aligned}
H_{\omega}(u)=\sum_{i=0}^{n} K_{i} \int_{\omega_{1}}^{\omega}\left(e^{\omega^{\alpha}}-e^{x^{\alpha}}\right)^{r-1} e^{x^{\alpha}} x^{\alpha-1+n-i} d x \\
\cdot \int_{u}^{1}(t-u)^{n-\beta-1} \frac{\sin (x t-a)}{t^{i+1}} d t .
\end{aligned}
$$

Now

$$
\int_{1}^{\infty}(t-u)^{n-\beta-1} \frac{\sin (x t-a)}{t^{i+1}} d t=O\left\{(1-u)^{n-\beta-1} x^{-1}\right\} .
$$

${ }^{7}$ L. S. Bosanquet [1]. 
It follows from a change of variable, the second mean value theorem, and a theorem on the $\Gamma$ function, ${ }^{8}$ that

$$
\begin{aligned}
\int_{u}^{\infty} & (t-u)^{n-\beta-1} \frac{\sin (x t-a)}{t^{i+1}} d t \\
& =u^{n-\beta-i-1} \int_{0}^{\infty} v^{n-\beta-1} \sin \left(x^{u}(1+v)-a\right) d v+O\left(u^{n-\beta-i-2} x^{-1}\right) \\
& =O\left(u^{-i-1} x^{\beta-n}\right)+O\left(u^{n-\beta-i-2} x^{-1}\right) .
\end{aligned}
$$

The lemma is proved by (11), (12), (13) and an easy estimate of the term $i=n$ in (11).

LEMMA 2. For $\omega>K$ and $0<u<1$,

$$
\begin{aligned}
H_{\omega}(u)= & \sum_{i=0}^{n} O\left(e^{\tau \omega^{\alpha}} u^{-\tau-i-1} \omega^{\tau(\alpha-1)+\beta-i}\right)+O\left(e^{(\tau-1 / 2) \omega^{\alpha}} \omega^{k_{1}} u^{-k_{2}}\right) \\
& +\sum_{i=0}^{n} O\left(e^{\tau \omega^{\alpha}} u^{n-\beta-\tau-i-1} \omega^{(\tau-1)(\alpha-1)+n-i-1}\right) \\
& +O\left(e^{\tau \omega^{\alpha}} \omega^{(\tau-1)(\alpha-1)+n-1}(1-u)^{n-\beta-1}\right) .
\end{aligned}
$$

Proof. From (13) we get

$$
\begin{aligned}
H_{\omega}(u)= & \sum_{i=0}^{n} \sum_{j=1}^{\tau} \sum_{p=0}^{r-1} K_{i j p} e^{(\tau-j) \omega^{\alpha}} \int_{\omega_{1}}^{\omega} e^{j x^{\alpha}} x^{c} d x \\
& \cdot \int_{u}^{1}(t-u)^{n-\beta-1} \frac{\sin (x t-b)}{t^{i+\tau}} d t+O\left(e^{(\tau-1 / 2) \omega^{\alpha}} \omega^{k_{1}} u^{-k_{2}}\right),
\end{aligned}
$$

and

$$
\begin{aligned}
\int_{u}^{1}(t-u)^{n-\beta-1} & \frac{\sin (x t-b)}{t^{i+r}} d t \\
= & u^{n-\beta-i-\tau} \int_{0}^{\infty} v^{n-\beta-1} \sin \{x u(1+v)-b\} d v \\
& +O\left((1-u)^{n-\beta-1} x^{-1}\right)+O\left(u^{n-\beta-i-r-1} x^{-1}\right) \\
= & u^{-\tau-i} x^{\beta-n} \Gamma(\beta-n) \sin \left(x u-b^{\prime}\right) \\
& +O\left((1-u)^{n-p-1} x^{-1}\right)+O\left(u^{n-\beta-i-r-1} x^{-1}\right) .
\end{aligned}
$$

From (14) and (15), Lemma 2 follows.

\footnotetext{
${ }^{8}$ E. C. Titchmarsh [5, p. 107].
} 
Proof of the theorem. By Lemma 1 and (2)

$$
\int_{0}^{\omega^{\alpha-1}} \phi_{\beta}(u) H_{\omega}(u) d u=o\left(e^{\tau \omega^{\alpha}}\right) \quad \text { as } \omega \rightarrow \infty
$$

and by (2) and Lemma 2

$$
\int_{\omega^{\alpha-1}}^{1} \phi_{\beta}(u) H_{\omega}(u) d u=o\left(e^{\tau \omega^{\alpha}}\right) \quad \text { as } \omega \rightarrow \infty
$$

By (9), (16), and (17), then,

$$
C_{\tau}(\omega)=s e^{\tau \omega^{\alpha}}+o\left(e^{\tau \omega^{\alpha}}\right) \quad \text { as } \omega \rightarrow \infty
$$

Thus the theorem is proved.

\section{REFERENCES}

1. L. S. Bosanquet, On Abel's integral equation and fractional integral, Proc. London Math. Soc. (2) vol. 31 (1930) pp. 134-143.

2. G. H. Hardy and J. E. Littlewood, New convergence criteria for a Fourier series, Annali Scuola Normale Superiore, Pisa (2) vol. 3 (1934) pp. 43-62.

3. G. H. Hardy and M. Riesz, The general theory of Dirichlet series, 1912, Cambridge.

4. G. H. Hardy, An extension of a theorem on oscillating series, Proc. London Math. Soc. (2) vol. 12 (1913) pp. 174-180.

5. E. C. Titchmarsh. The theory of functions, 1932.

6. F. T. Wang, On Riesz summability of Fourier series, Proc. London Math. Soc. (2) vol. 47 (1942) pp. 308-325.

National University of Chemiang 